\title{
Advanced Observation and Telemetry Heart System utilizing Wearable ECG device and a Cloud Platform
}

\author{
Ioannis Chatzigiannakis ${ }^{1}$, Emil Stoyanov Valchinov ${ }^{1}$, Athanasios Kalogeras ${ }^{1,2}$, Christos Alexakos ${ }^{1}$, Panagiotis \\ Konstantinopoulos ${ }^{1}$ \\ ${ }^{1}$ Computer Technology Institute and Press Diophantus (CTI), Patras, Greece \\ ${ }^{2}$ Industrial System Institute/ATHENA Research Center (ISI), Patras, Greece \\ \{ichatz,kalogeras,alexakos,konstantin\}@cti.gr, valchinov@gmail.com
}

\begin{abstract}
Short lived chest pain episodes of post PCI patients represent the most common clinical scenario treated in the Accidents and Emergency Room. Continuous ECG monitoring could substantially diminish such hospital admissions and related ambulance calls. Delivering community based, easy-to-handle, easy to wear, real time electrocardiography systems is still a quest, despite the existence of electronic electrocardiography systems for several decades. The PATRIOT system serves this challenge via a 12-channel, easy to wear, easy to carry, mobile linked, miniaturized automatic ECG device and a Cloud platform. The system may deliver high quality electrocardiograms of a patient to medical personnel either on the spot or remotely both in a synchronous or asynchronous mode, enhancing autonomy, mobility, quality of life and safety or recently treated coronary artery disease patients.
\end{abstract}

Keywords- eHealth; electrocardiograph; ECG; telemetry; telemedicine; cloud

\section{INTRODUCTION}

Percutaneous Coronary Intervention (PCI or "stent placement") in the context of balloon Angioplasty is the main way of treating coronary artery disease. This corresponds to opening, or dilating the lumen of a narrowed coronary artery, with a balloon or a stent, restoring the blood and oxygen supply to the heart. However recent trials state an increased prevalence of angina (chest pain) after successful PCI, as high as $30 \%$, one year following the procedure [1].

The most common clinical scenario treated in the Accidents and Emergency Room, reflects to a recently (within one year) treated coronary artery disease patient, managed with either a stent intervention (PCI), or by-pass operation, having a short lived (less than 5 minutes) chest pain episode [2]. The patient usually calls for help in the first instance of chest pain. The ambulance crew collects the patient, transfers him/her to the nearest hospital, and performs some tests on the way. In most cases (ambulance calls, emergency admission, admission through family doctor), an admission electrocardiogram (an electrical heart trace) is used as the gold standard to identify and/or rule out the presence of life threatening ischemia i.e. newly appearing lack of blood or oxygen in the recently treated

This work was supported by the Greek, nationally funded, research project "PATRIOT" -NSRF 2007-2013, Call: Cooperation 2011. heart. The new admission electrocardiogram (ECG) is looked at and compared to the most recent one (e.g. the one the patient had at the discharge from the hospital, following one's coronary artery opening treatment). As a rule of thumb, if the electrocardiogram is positive for new abnormal electrical changes the patient is admitted and treated. Vice versa if the electrocardiogram is negative for ischemic changes, the patient is most times discharged and followed up on outpatient basis, unless the symptoms persist. Therefore the ECG trace during a patient's angina episode is of high predictive value to either confirm, or exclude a new heart attack. The vast majority of admitted patients within one year post PCI, lack hospital admission ECG evidence that would substantiate their admission and therefore most patients get discharged within $24 \mathrm{H}$ following their ambulance call.

Although ambulance crew usually reach the post-PCI angina patient within one hour post angina claim for hospitalization, and the ECG is usually normal upon arrival, the patient is almost always admitted to the Coronary Care Unit for a closer check. This is mainly due to the fact that normal ECG, performed upon ambulance's arrival is not sufficient to preclude an abnormal ECG at an earlier moment, when the patient was claimed symptomatic. Such hospital admissions could theoretically be substantially diminished by continuous ECG monitoring to specific patient groups. Yet, this has not been implemented in clinical practice, mainly due to such ordinary telemedicine obstacles as : lack of friendly - easy to use devices from e.g. such groups as elderly patients, big size of devices, lack of cost effectiveness, and absence of land line communication system in most of the times.

In view of the above necessity, the present paper presents the PATRIOT system - PATRas Instant Observation and Telemetry heart system. The first main objective of PATRIOT is to deliver a cost effective, $\mathrm{Wi}-\mathrm{Fi}$, mobile based, community based, easy to handle, miniature, easy to wear, ECG monitoring system that provides real time ECG trace on demand to the cardiac frequently-admitted patient up to one year post a patient's coronary treatment. This system serves the purpose of providing enhanced autonomy, mobility, safety, reassurance and quality of life to the post-PCI patient, though real time electrocardiography, eliminating the patient's fear of death and significantly reducing unnecessary hospitalizations and ambulance calls. The PATRIOT system makes possible 
real time electrocardiography utilizing miniature mobile devices capable of identifying, flagging up and automatically sending an alert signal through the patient's mobile phone, in the case that a life threatening ECG change is encountered.

Chapter II of the paper presents the relevant state-of-art about wearable ECG devices and integrated e-Health systems. Chapter III of the paper presents the proposed PATRIOT system, in terms of its general architecture and its individual components. Chapter IV of the paper briefs the use case scenarios of the PATRIOT system. Finally, some conclusions are drawn in chapter IV.

\section{RELATED WORK}

Although electronic electrocardiography systems exist in the form of recording diagnostic devices for more than three decades, it has not been possible to date to deliver community based, easy-to-handle, easy to wear, real time electrocardiography systems. This is mainly attributed to the following:

1) Signal Quality: A good signal quality is based on and requires accurate clearance and/or inhibition of electromagnetic interference. Electromagnetic noise may be either of environmental (e.g. mobile phones, electrical wires, appliances) or internal origin (e.g. produced by muscular contractions) and remains a frequent source of abnormally appearing ECGs, encountering for repeat test or false positive test [3].

2) Battery and memory capacity: Good signal quality, and lengthy ECG recordings require battery consumption and memory capacity [4]. This becomes increasingly more demanding as more than one ECG channels (meaning electrical depolarization recording points) are used. It is usual that more than one recording points (three, six, twelve) are needed in order to acquire more information from more than one heart regions. Each recording point (so called ECG lead) increases proportionally the battery and memory demand of the device. Most of the new electrocardiographers are capable of providing accurate diagnostic ECG recordings, while their diagnostic use has been limited to $24 \mathrm{~h}$ monitoring due to power and memory technology limitations. Although weekly, monthly and implantable six-monthly continuous ECG recording devices are commercially available, they correspond to proportionally higher battery and memory demand, bigger size and price, reflecting reversibly to signal quality and a cost rising in logarithmic pattern, ranging from 2,000 Euros up to 10,000 Euros.

3) Real time recording and synchronous transmission: $24 \mathrm{~h}$ High quality ECG recording (so called Holter) devices are usually reserved for the cases where an unexplained cardiac symptom, such as a cardiac arrhythmia, or chest pain, needs to be investigated [5]. As aforementioned good signal quality recording impacts on the battery consumption. Standard mobile phone $\sim 1000 \mathrm{mAh}$ batteries usually suffice marginally for $18 \mathrm{~h}$ 3-channel moderate quality ECG recording. Therefore additional Wi-Fi transmission to a nearby receiver would substantially exhaust the power supply and limit significantly the recording time. It becomes apparent that this would render the real time ECG device to an ineffective short-lived transmitter without any clinical impact on the patient's wellbeing.

The design of electrocardiogram devices has followed closely the electronics miniaturization evolution over the years [6][7]. During the last decade several miniature ECG recording systems have evolved. In clinical practice Medtronics Inc, occupying for many years the highest proportion of the market, launched first the implantable REVEAL Implantable transmitters in 2000 and followed this evolution by the development in July 2011 of a mobile application Wi-Fi alert system linked to an implantable ECG recorder, namely CareLink. Miniature nonclinical loop recorders emerged in 2005 even for $35 \mathrm{~g}$ rodents to cover the ECG needs of laboratory cardiology (ADinstruments-mouse ECG transmitter). Several of these systems were linked to proprietary technology and mathematical algorithms for ECG abnormalities detection. Furthermore, Corventis announced the external AVIVO system operating also in automatic (autodetection and transmission) mobile mode. One non-clinical iPhone application converting one's iPhone to ECG (Alivecor iPhonECG) has also emerged since 2010.

However, to our knowledge, neither of these systems has either clinically proven cost effectiveness, or a clinically proven predictive value (positive or negative) for ischemic heart disease follow up and alert. The reason is threefold. At first, more than one ECG channels and good signal quality is necessary to provide an ischemic trace of significant predictive value. In most clinical cases, the aforementioned loop ECG devices are expensive, implantable under the skin providing a single channel ECG trace; therefore they are reserved solely for a minority of arrhythmic undiagnosed patients, needing ECG supervision and occasionally alert, as opposed to ischemic heart disease follow up. Moreover in the cases where nonimplantable ECG recording devices are used for the same purpose, the electronic interference and signal quality disturbances e.g. from pectoralis muscular contractions, have not delivered a clinically useful cost-effective result quite as yet. At second, a low cost ( $<570$ Euros) system is required to deliver a device able to compensate cost-effectively the routine emergency admission costs. At third, to our knowledge, - no 6channels, easy to wear, easy to carry, mobile linked, automatic ECG technology exists in the market capable of serving this purpose.

\section{PATRIOT SYSTEM}

\section{A. General Arcitecture}

PATRIOT aims at delivering high quality electrocardiograms from a wearable ECG to medical personnel at a remote location (office or hospital/clinic). The proposed system architecture can be seen in Figure 1. The ECG wearable device is attached to the patient's body and its data is transmitted to the patient's smartphone. The mobile phone application is responsible for two functionalities: a) to offer a Helioseismic and Magnetic Imager (HMI) for the presentation of the recorded electrograph to the patient in real-time and b) to upload ECG data to the PATRIOT Cloud Platform. The smartphone's capability to transmit data to the Internet utilizing either Wi-Fi or $3 / 4 \mathrm{G}$ available connections, allows the 


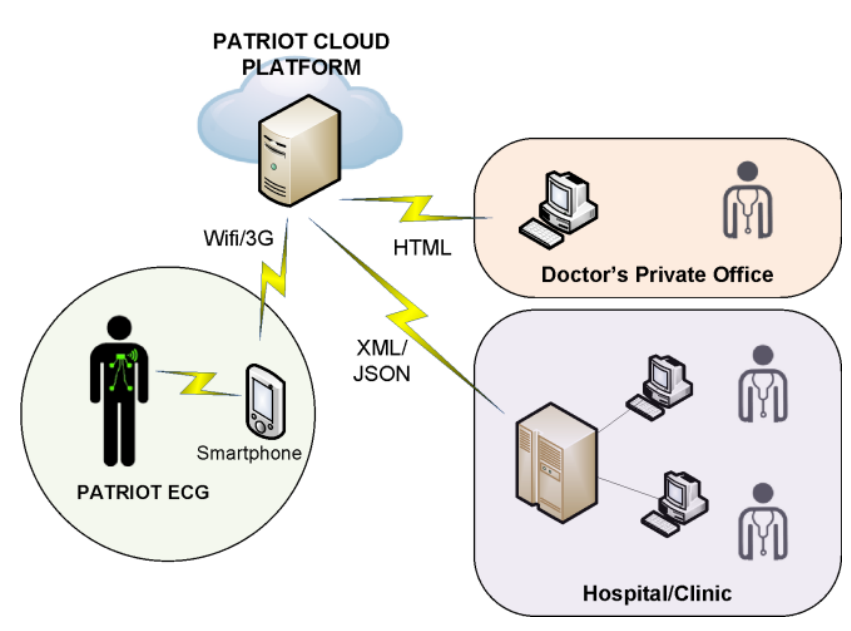

Fig. 1. PATRIOT System Architecture

PATRIOT system to be fully functional in case of usage both in static places such as the home/office environments and in moving environments such as an ambulance during submission. The ECG trace is stored on the PATRIOT Cloud platform according to the European Data Format (EDF+) specifications. The responsible medical personnel e.g. a doctor can have access to the ECG trace at his device via a Graphical User Interface which is based on the HTML standard for interaction through a web browser (IE, Google Chrome, Firefox, Safari, etc.). Furthermore, a web service interface between the PATRIOT Cloud Platform and the Hospital Information System can serve the purpose of integrating PATRIOT's data to the hospital legacy systems. The recording sessions may be also optionally stored on the ECG device in a compressed format and can be recovered on demand at any time.

\section{B. Wearable ECG}

The PATRIOT ECG device enables a light weight 6channel ECG Wi-Fi tool, aiming at preventing from the cost effectiveness point of view unnecessary post-PCI admissions, on the basis of its low cost and accurate ECG recording. Moreover its light wearability, signal accurateness and easy battery-charge or change capability makes it a very competitive external ECG recorder, able to challenge very effectively even existing implantable automatic loop recorders.

Advanced compact integrated hardware and software tools have been developed and used in order to deliver a commercially competitive ECG monitoring system with a three-fold range of clinical applications. First of all, smart hardware and software was used in order to achieve power saving. Secondly, a system was designed with the capability of real time recording and long distance transmission by contemplating and incorporating a buffering filter. In brief this system corresponds to a memory buffer that at first stores the ECG trace, then it sends it to a software application in a smartphone which utilizes mathematical algorithms that only store the abnormal or potentially abnormal ECG traces, while discarding the rest, and finally saves and transmits only the suspicious ECG signals to the on call, or attending near-by medical facility via the patient's mobile phone, through a user friendly automatic mobile interface. In parallel size and weight deduction, down to $70 \mathrm{~g}$, was achieved, while wearability was eased by splitting the electronic board in four compartments.

The PATRIOT device wireless main unit comprises a 32bit high-performance MCU (microcontroller), $2.4 \mathrm{GHz}$ radio and flash memory. The latter is implemented with the $\mathrm{Wi}-\mathrm{Fi}$ Transceiver Module MRF24WG0MA (Microchip). This module provides a comprehensive solution with included RF components, integrated PCB antenna, simple four-wire SPI interface and full featured TCP/IP protocol stack. The amplified ECG signals are digitized by the embedded 10-bit ADC. The local data storage is implemented with 4Gbit (512Mx8bit) NAND Flash memory.

The system is powered by a rechargeable coin cell LithiumIon polymer battery, which is the optimal choice in terms of dimensions and weight. However Li-ion batteries require more precise charging procedures so a dedicated battery charger is used in order to prevent the battery voltage from rising above 4.2 volts. Power supply regulation is implemented with two $3.3 \mathrm{~V}$ low noise low-dropout integrated linear regulators: one for the analog part - biopotential amplifier, and another for the digital part - the microcontroller and the radio transceiver. This arrangement aims at minimizing the disturbances in the analog circuitry caused by the higher current consumption of the radio transceiver. Biopotential amplifiers working with bipolar signals require dual power supplies for proper operation. In order to create a balanced power supply, a virtual reference common was used at $1.25 \mathrm{~V}$, implemented with a precision, low power, low dropout integrated voltage reference.

The overall design aims at minimizing the motion artifacts, the power line interference, the interference between the analog and digital part of the circuit and the size of the system, thus achieving high signal quality. The ECG device is capable of operating in 3 basic modes:

- HOLTER

- miniHOLTER

- real-time.

In the HOLTER mode, the device records ECG data until the memory is full, or the maximum number of allowed recorded sessions is reached, or an explicit command to stop the recording is issued. The device can operate in offline energy saving mode, while the recording is ongoing. Additionally the push button on the device can be used to switch on or off the wireless connectivity of the device.

In the miniHOLTER mode, the device records ECG data, on demand, for a specified duration. The recording can be interrupted if an explicit command to stop the recording is issued. The device can operate in offline energy saving mode, while the recording is ongoing.

In the real-time mode, the device records ECG data only while connected to a smartphone device, and only for the duration of an explicitly initiated connected session. The realtime mode can optionally save data in the memory available on the device, to allow for more reliable communication with the 


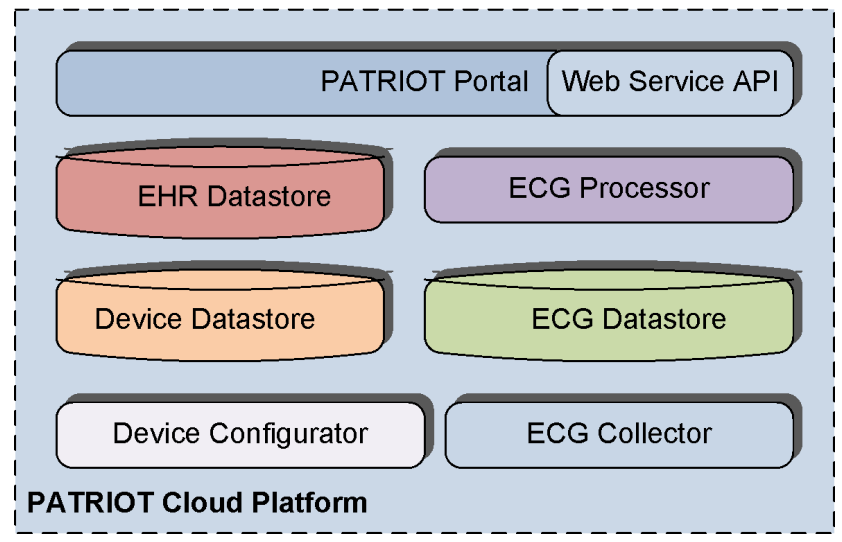

Fig. 2. PATRIOT Cloud Platform Components

device and provide the additional capability to recover the recorded ECG data session at a later time.

Moreover, the device is capable of transmitting the data of specific sessions to a smartphone, while recording another session in HOLTER and miniHOLTER modes. The maximum allowed number of recorded ECG data sessions is 255. The ECG device supports ECG data recordings with variable settings for the ECG channels and sampling rate. The allowed values for channel number are 6 and 12, and the allowed sampling rates are $400 \mathrm{~Hz}, 500 \mathrm{~Hz}, 800 \mathrm{~Hz}$ and $1 \mathrm{KHz}$.

The following table shows the maximum duration of an ECG recording that the ECG device is capable of sampling for typical configurations.

TABLE I. PATRIOT ECG BATTERY LIFE MEASUREMENTS

\begin{tabular}{|c|c|c|}
\hline Channels number & Sampling Rate & Duration \\
\hline 12 & $1 \mathrm{Khz}$ & 14.9 hours \\
\hline 12 & $500 \mathrm{hz}$ & 29.81 hours \\
\hline 6 & $1 \mathrm{Khz}$ & 59.62 hours \\
\hline 6 & $500 \mathrm{hz}$ & 119.24 hours \\
\hline
\end{tabular}

\section{PATRIOT Cloud Platform}

The PATRIOT Cloud Platform is an integrated system responsible for the management of the registered ECG devices, the ECG data collection and processing, the patient's clinical data storage and the delivery to the doctors of the collected data. The PATRIOT's Cloud Platform main functional components are depicted in Fig. 2.

The PATRIOT Portal is the central orchestrator for all the services that the platform provides. Its users (doctors as well as patients or individuals) may access it at any time to go through the medical data stored in it, using their PCs or smartphones through a unified interface. Furthermore, it provides a Web Service API that enables quick integration of the platform with third-party applications such as the Hospital Information Systems. The core data of the PATRIOT portal is stored in a semantically rich storage for entities and relationships.
The ECG Collector component provides the interface to the smartphone connected with the PATRIOT ECG, permitting the latter to transmit data to the platform. The data is stored in the ECG Datastore component. It is also responsible for accessing the ECG traces and providing them to either the web portal or to other services of the PATRIOT platform for on demand processing and analysis. Furthermore, it provides the required caching, compression and decompression mechanisms to facilitate and speedup the communication between the different PATRIOT components.

The Device Configurator acts as a gateway between the ECG devices and the Web Portal. Due to the fact that the devices are not always connected to the network, it is not always possible to configure them in real time. Whenever the devices are connected to the network any configuration changes (such as the mode of data acquisition) are automatically pushed down to the devices. When the devices are not connected to the network the new settings are stored in the Device Datastore. Whenever devices find connectivity, they query the Device Datastore, receive the latest settings and henceforth operate based on them

The EHR Datastore component acts as a storage service for a patient's complete medical history (Electornic Health Record), containing information either acquired through the ECG devices or via the in-person interaction between the patient and the doctor.

The ECG Processor operates continuously in the background and extracts information (like heart rate) from received ECG traces. The information extracted can be then used to notify the doctors for the status of their patients. The ECG Processor forwards the detected events to the portal in order to notify the doctor. This can be achieved taking into account the severity of the information by using email, push notifications or, if needed, voice and SMS messages to the doctor's mobile phone.

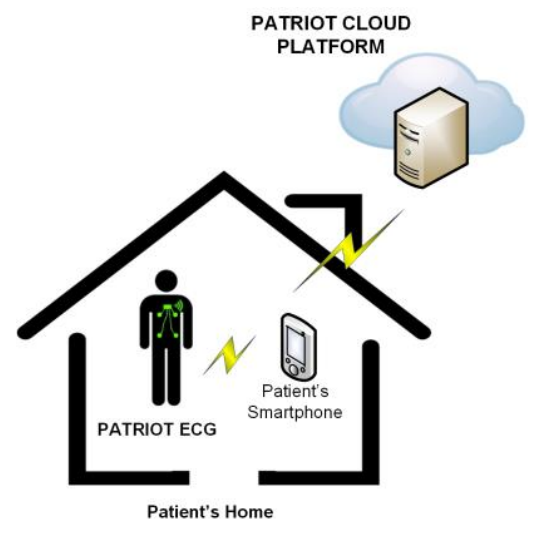

Fig. 3. Self-Monitoring

\section{USE CASE SCENARIOS}

The PATRIOT system design envisions covering five major usage scenarios. The first scenario (scenario 1) targets end-users that would like to perform self-monitoring of their medical conditions. These users might be either patients that 


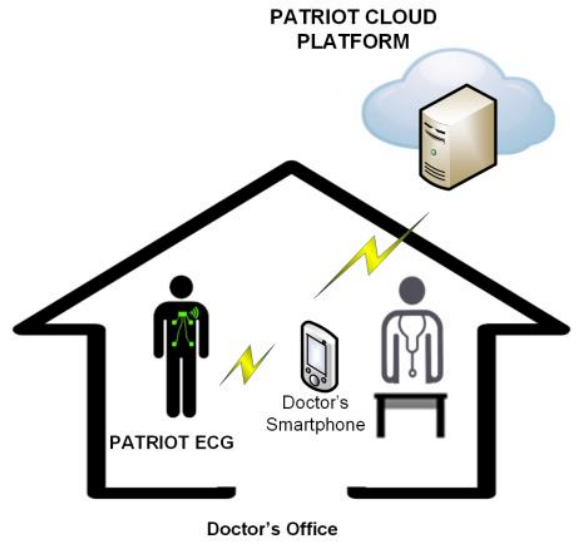

Fig. 4. Doctor monitoring a Patient on the office

have recuperated from a heart incident, which they need to monitor themselves periodically, or individuals that are aware about their medical condition such as athletes, people that have a cardiac record in their family history etc. The second scenario (scenario 2), doctors are targeted that will utilize the PATRIOT system to monitor their patients either at their office or at a patient's home during a visit. The third and fourth scenarios (scenario 3 and 4) represent extensions of the second, where the patients carry the ECG device at home in the holter mode and after a period of time return the device back to the doctor to have the ECG traces checked (scenario 3), or where the patients using also their smartphones store the ECG traces to the main platform (scenario 4). The final scenario (scenario 5) utilizes the PATRIOT system inside an ambulance or a hospital and then the ECG devices are operated by a doctor or a nurse who instantly acquire the ECG traces of the patient, and monitor in real time the data that is stored in the main platform.

\section{A. Scenario 1: Self-Monitoring}

In scenario 1, the PATRIOT system serves self-monitoring purposes (Fig. 3). At first, the user switches-on the PATRIOT ECG. Afterwards, the user uses a PATRIOT application in one's smartphone for detecting the device in the local network. Upon the device discovery, the user creates an account on-line in the PATRIOT Cloud Platform and pairs the ECG device so as to monitor oneself. Then, the system is ready to use. The acquired ECG traces are relayed through the user's smartphone

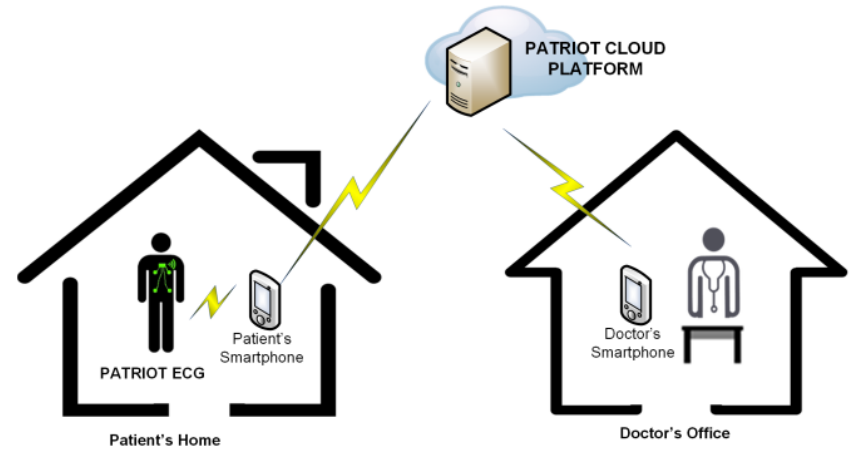

Fig. 5. Doctor remote and synchronous monitoring of a Patient

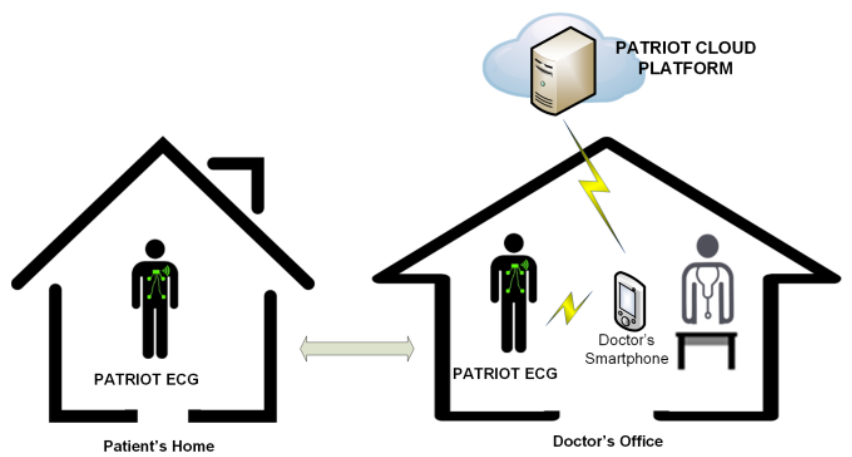

Fig. 6. Doctor remote and asynchronous monitoring of a Patient

application to the ECG Collector that processes them for storage to the PATRIOT Cloud Platform. The smartphone device can also provide visualization of the data that is being relayed. The streamed data is not stored locally; a connectivity service can be employed to resolve intermittent connectivity issues. The user can view reports and search traces remotely after they are synced with the server. Asynchronous ECG alerts are forwarded to the user's smartphone application. Furthermore, if the patient becomes symptomatic (e.g. experiences chest pain), the patient wears the ECG device, calls at the hospital and the same time activates the ECG acquisition and transmission service. The scenario 5 then is activated.

\section{B. Scenario 2: Doctor monitoring a Patient at the infirmary}

This scenario fits to the occasions when the patient visits a doctor's infirmary (Fig. 4). The same scenario is activated, when doctor visits the patient at home. The doctor may setup the ECG device using one's smartphone following the same first steps as in scenario 1 . The doctor, may configure a new profile - if not existent - for the patient through one's own account on the PATRIOT Cloud Platform. The PATRIOT ECG is configured to the miniHOLTER mode and it is worn by the patient. The ECG traces are relayed through the Doctor's smartphone Application to the PATRIOT Cloud Platform. The smartphone device may also provide visualization of the data that is being relayed. The streamed data is not stored locally; a connectivity service can be employed to resolve intermittent connectivity issues. The doctor may view reports, search traces and examine ECG alerts aggregated on the Patient's profile remotely after they are synced with the PATRIOT Cloud Platform.

\section{Scenario 3: Doctor records a patient's ECG using a Holter mode}

In this scenario (Fig. 5), the patient visits the doctor at his infirmary, the doctor sets up the PATRIOT ECG and gives it to the patient. The ECG device is paired with the doctor's smartphone and a patient profile is created on the PATRIOT Cloud Platform following the same procedure as in scenario 2. The ECG is configured in the Holter mode. The patient wears, turns on the ECG device and returns home. After the ECG's memory is full, the patient comes back to the doctor where through the doctor's smartphone, the ECG trace is uploaded to the PATRIOT Cloud Platform. The doctor can view reports, 
search traces and examine ECG alerts remotely after the synchronization with the PATRIOT Cloud Platform.

\section{Scenario 4: Doctor remote and synchronous monitoring of a Patient}

This scenario is very similar to the previous scenario 3 except that the doctor may monitor in real-time the ECG trace of a patient wearing the device at one's home (Fig. 6). In this case the ECG is setup to interoperate with the patient's smartphone as presented in the previous scenarios. The doctor sets the ECG device to operate in real-time mode. The Doctor records ECG data on demand by activating the ECG device either in real-time mode or in the Holter mode remotely. The ECG traces are relayed through the patient's smartphone application to the PATRIOT Cloud Platform. Asynchronous ECG alerts are forwarded to the doctor's smartphone Application. The doctor can view the patient's recorded data through the web portal of the PATRIOT Cloud Platform.

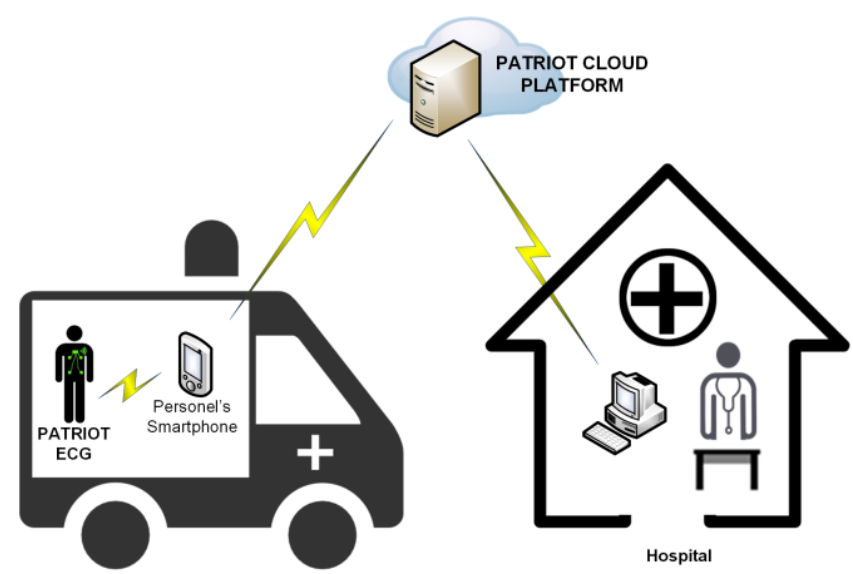

Fig. 7. Patient Monitoring in Hospital/Ambulance

\section{E. Scenario 5: Patient Monitoring at the Hospital/ in the Ambulance}

This scenario (Fig. 7) is referred to occasions of emergency submission of a patient with chest pain to a hospital. In this case the ECG devices are pre-configured with medical staff's smartphones and the associated accounts are created in the PATRIOT Cloud Platform. Furthermore, the ECG devices are set to communicate either via a dedicated gateway (various $\mathrm{Wi}$ Fi access points in the Hospital's network, e.g. per floor). In the case of using the ECG device in an Ambulance, a gateway device in the Ambulance can be a Wi-Fi Access Point for the ECG device to connect to, which communicates with the Hospital network via 3/4G. The dedicated gateways act as relays. They also register themselves in the cloud platform. In the case of an emergency incident the ambulance's personnel wears the ECG device to the patient and turns it on. For reason of time-saving, a temporary patient profile is created on the PATRIOT Cloud Platform by the personnel's smartphone. The
ECG trace is uploaded on the PATRIOT Cloud Platform using the various devices (smartphones, Hospital's Wi-Fi Access Points) that act as gateways along site to the route from the point of the patient collection to the Emergency Room. Doctors are able to monitor or view the ECG trace through the Hospital Information System that is integrated with the PATRIOT Cloud Platform.

\section{CONCLUSION}

The paper presents the PATRIOT system, a system aiming at delivering high quality electrocardiograms from a wearable ECG device to medical personnel at a remote location (doctor's infirmary or hospital/clinic). The architecture of the system is presented and its sub systems are analyzed. Five use case scenarios are also presented that indicate the system's potential usage for patient self-monitoring, doctor monitoring of a patient either on site or remotely, both synchronously or asynchronously, and medical personnel monitoring of a patient at a hospital or in an ambulance during submission. The presented system offers a 12-channel, easy to wear, easy to carry, mobile linked, automatic, miniaturized ECG device, that may provide enhanced autonomy, quality of life, mobility and safety to the post-PCI patient, while significantly reducing unnecessary hospitalizations and ambulance calls. Future work relates to the clinical testing of the system and its utilization according to the specified use case scenarios.

\section{REFERENCES}

[1] Huqi A, Morrone D, Capati E, Urselli R, Marzilli M. PREVALENCE OF ANGINA AFTER SUCCESSFUL PCI. J Am Coll Cardiol. 2010;55(10s1):A128.E1201.

[2] Koonlawee Nademanee, Vanida Intarachot, Pramil N. Singh, Martin A. Josephson, Bramah N. Singh, Characteristics and clinical significance of silent myocardial ischemia in unstable angina, The American Journal of Cardiology, Volume 58, Issue 4, 15 August 1986, Pages B26-B33, ISSN 0002-9149.

[3] Baranchuk Adrian, Kang Jaskaran, Shaw Cathy, Campbell Debra, Ribas Sebastian, Hopman Wilma M., Alanazi Haitham, Redfearn Damian P., Simpson Christopher S., "Electromagnetic Interference of Communication Devices on ECG Machines", Journal of Clinical Cardiology, Vol. 32, Issue 10, pp. 588-592, Wiley Periodicals, Inc., 2009, SN: 1932-8737

[4] Fariborzi, H.; Moghavvemi, M.; Mehrkanoon, S., "Design of a Lowpower Microcontroller-based Wireless ECG Monitoring System," Research and Development, 2007. SCOReD 2007. 5th Student Conference on , vol., no., pp.1,4, 12-11 Dec. 2007

[5] Crawford M, Bernstein S, Deedwania P, et al. ACC/AHA guidelines for ambulatory electrocardiography: A report of the American College of Cardiology/American Heart Association Task Force on Practice Guidelines, J Am Coll Cardiol. 1999;34(3):912-948.

[6] Jeon, Byungkook, Jundong Lee, and Jaehong Choi. "Design and implementation of a wearable ECG system." International Journal of Smart Home 7.2 (2013): 61-69.

[7] Nemati, Ebrahim, M. Jamal Deen, and Tapas Mondal. "A wireless wearable ECG sensor for long-term applications." Communications Magazine, IEEE 50.1 (2012): 36-43 